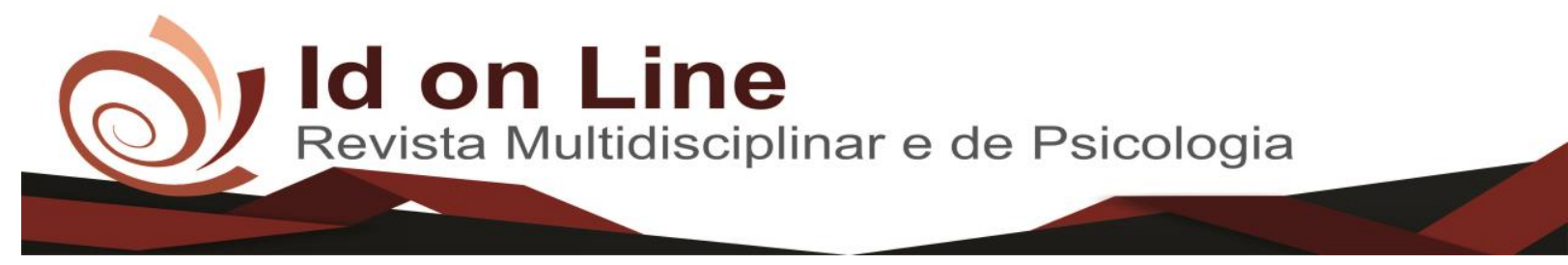

Artigo

\title{
A Gestão da Cadeia de Suprimentos e o Nível de Inovação: Estudo de Caso nas Indústrias de Calçados na Cidade de Juazeiro do Norte - Ceará
}

\author{
José de Figueiredo Belém ${ }^{1}$; José Eduardo de Carvalho Lima²; \\ Josiano Cesar de Sousa ${ }^{3}$ :Murilo Barros Alves ${ }^{4}$
}

\begin{abstract}
Resumo: O objetivo deste artigo é analisar os fatores essenciais da gestão da inovação em empresas do ramo de fabricação de calçados na cidade de Juazeiro do Norte - Ceará. A principal técnica de pesquisa foi entrevistas semi-estruturadas com gestores das empresas pesquisadas. No estudo foi desenvolvido um quadro teórico para as práticas de inovação em indústrias, formado por dois tipos: Inovação de produtos e inovação de processos. O estudo investiga esses dois tipos de inovação em três grandes empresas que atuam em forma de clusters e concorrem no mesmo mercado. É abordado o tema dos modelos de inovação e sua evolução ao longo do tempo, seus diversos estágios, sequências, interfaces, interações e interferências nas empresas e sistema de Gestão da Cadeia de Suprimentos. Observou-se durante a investigação a falta de adequação dos processos em alguns requisites importantes e consequentemente os produtos não atendem aos requisitos essenciais de inovação, tornando-se necessário que as empresas se aprofundem nos conhecimentos sobre a inovação e os apliquem corretamente a seus negócios, com o propósito de aumentar a competitividade e maximizar seus resultados.
\end{abstract}

Palavras Chave: Inovação, Gestão, Cadeia de suprimentos.

\section{Supply Chain Management and the Level of Innovation: A Case Study in the Shoe Industries in the City of Juazeiro do Norte, State of Ceará}

\begin{abstract}
The objective of this article is to analyze the essential factors of innovation management in companies of the footwear manufacturing sector in the city of Juazeiro do Norte - Ceará. The main research technique was semi-structured interviews with managers of the companies surveyed. In the study, a theoretical framework was developed for the innovation practices in industries, formed by two types: Product innovation and process innovation. The study investigates these two types of innovation in three large companies that act in the form of clusters and compete in the same market. It addresses the theme of innovation models and their evolution over time, their various stages, sequences, interfaces, interactions and interferences in companies and the Supply Chain Management system. During the investigation, the lack of suitability of the processes was observed in some important requirements and consequently the products do not meet the essential requirements of innovation, making it necessary for companies to deepen their knowledge about innovation and apply them correctly to their businesses, with the purpose of increasing competitiveness and maximizing its results.
\end{abstract}

Keywords: Innovation, Management, Supply Chain.

\footnotetext{
${ }^{1}$ Doutorando em Engenharia de Produção e Sistemas pela Universidade do Vale do Rio do Sinos - UNISINOS, Rio Grande do Sul. Mestre em Economia pela Universidade Federal do Ceará, Graduação em Administração Geral pela UNILEÃO. josebelem@ @eaosampaio.edu.br; ${ }^{2}$ Graduação em Matemática pela Universidade Regional do Cariri, Mestrado em Economia do Setor Público pela Universidade Federal do Ceará e Doutorando em Engenharia de Produção e Sistemas pela Universidade do Vale do Rio dos Sinos. Professor titular do Centro Universitário Dr. Leão Sampaio e Professor da Faculdade Paraíso. Contato: Eduardo.doutoradounisinos@ gmail.com;

${ }^{3}$ Doutorando em Engenharia de Produção e Sistemas, pela Universidade do Vale do Rio dos Sinos-UNISINOS, Mestre em Administração pela Universidade Católica de Salta - Argentina. Contato: josianocesar@hotmail.com;

${ }^{4}$ Doutorando em Engenharia de Produção pela Universidade Vale do Rio Sinos - RS. Mestre em Ensino da Matemática pela PUC-MG, Professor nível I da Devry Facimp e professor assistente I da Universidade da Região Tocantina do Maranhão (UEMASUL). Contato: muriloimp@yahoo.com.br.
} 


\section{Introdução}

A indústria de calçados brasileira está concentrada em grupos regionais. Este estudo aborda as empresas do ramo calçadista de Juazeiro do Norte - Ceará, na região do Cariri Cearense, onde estão concentradas mais de 200 unidades industriais do ramo, competindo no mesmo mercado, sendo que $3 \%$ são de grande porte, $22 \%$ de médio e o restante $(75 \%)$ são pequenas Segundo o (Sindindústria 2017).

As indústrias funcionam de forma mista, ou seja, algumas associadas e as demais de forma isolada, portanto, caracterizando-se como clusters em uma parte das mesmas, sendo que as demais perdem a competitividade que teriam, de acordo com (Cooper, 2001).

O objetivo deste arigo é analisar os fatores essenciais da gestão da inovação em empresas do ramo de fabricação de calçados na cidade de Juazeiro do Norte - Ceará.

Para analisar o nível desenvolveu-se um quadro teórico de práticas inovadoras nas industriais já mencionadas na literatura procurando identificar quais as praticas as empresas adotam nessa região, e para discutir os resultados e compará-los com a revisão bibliográgica.

O restante do trabalho contém a revisão, a pesquisa, a discussão dos resultados e as considerações finais.

As atividades concentradas na região do mesmo ramo de indústrias geram vantagens competitivas como consequência de uma relação entre distâncias entre empresas e alta especialização nas atividades (Prause 2014; Vicente e Suire 2007). Porém a formação de clusters industriais ( $\mathrm{Li}$ et al. 2012) nesta região ainda não é bem concentrada, dificultando a coopetition, que pode gerar resultados influenciadores nas mais diversas formas de inovação.

Os interesses em inovação na área industrial, Segundo (Gunasekaran 1999) aumentaram nos últimos anos, tanto no meio acadêmico, como também nos gestores de Cadeias de suprimentos. A importância dada à inovação como um elemento de competitividade na economia global justifica esse interesse. Cinco abordagens teóricas ajudam a classificar a pesquisa sobre as indústrias. De acordo com os teóricos principais no assunto a inovação representa um meio poderoso de redução de custos de transação geralmente presentes na indústria.

De acordo com (Freeman; Soete, 1997) a inovação tem se transformado em meta para os mais diferentes tipos de organizações, sendo que em cada realidade, diversos aspectos devem 
ser observados a fim de estimular seu crescimento e eliminar as barreiras que podem dificultála. Trata-se de uma dificuldade imensa entre o que se projeta e o que se realiza, com diferentes maneiras de perceber as dimensões e contextos de aplicação que, como consequência, é compreendido sob diferentes formas de produção de conhecimento em vários campos, ramos de atividade e setores das indústrias, como por exemplo, as de calçados.

$\mathrm{Na}$ visão de (Campos 2016), a construção e o aclaramento do conceito de inovação reforçam a necessidade de busca por definição mais operacional abrindo um espaço para a organização e sistematização dos estudos propostos no presente trabalho.

Para (Betz, 1997) os princípios adotados bem como as ferramentas básicas de manufatura apresentam-se como forma adequada para qualquer tipo de empresa que pretende maximizar seus lucros no que tange a eficiência em sua produção adotando também práticas de inovação. Muito embora, de forma equivocada, haja percepção de que somente as grandes empresas implementam técnicas modernas de gestão da produção, com práticas inovadoras.

Atentando-se para (Westkamper, 2007), o grande desafio, porém, está na concepção dos gestores, para uma nova forma de administrar em se tratando de cadeia de suprimentos aliada ao processo de inovação, e adotar práticas direcionadas a racionalização dos procedimentos e meios de produção.

Segundo (Betz 1997), para o meio empresarial a inovação não pode ser encarada apenas como uma oportunidade de iniciar um negócio ou lançar um produto, crescer em um negócio existente ou mesmo sobreviver. Mas, uma maneira de definir os rumos da indústria, do negócio ou mesmo da comunidade onde a organização ou entidade está inserida. Contudo, toda vez que se trata do tema inovação, seja de um novo produto, um novo processo ou mesmo uma nova prática gerencial, depara-se com uma dificuldade de desenvolvimento e implementação.

Combinando com (Damanpour 1991), é que o presente trabalho está pautado na investigação a fim de que se possa constatar os fatores que motivam empresas do ramo de calçados no Cariri Cearense a inovar produtos e processos: indagar sobre a utilização de práticas que garantam a inovação e a competitividade; analisar as formas como são praticadas as políticas sob a ótica da inovação; pesquisar e descrever os modelos de gestão da inovação; apresentar as práticas de inovação conhecidas no mercado de calçados; e descrever a evolução das empresas no seu mercado. 


\section{Gerenciamento da Cadeia de Suprimentos}

Para (Fajnzylber 1988, p. 12), as empresas procuram combinar suas vantagens comparativas tradicionais com as vantagens competitivas mais duradouras e sustentáveis. Neste sentido, um aspecto essencial, é o papel central gerado pela inovação para a competitividade sustentável que contrasta, com a usual prioridade dada à exploração das vantagens competitivas tradicionais como baixo custo de mão-de-obra de exploração de recursos naturais.

O gerenciamento da cadeia de suprimentos segundo (Betz 1997) destaca as interações logísticas que acontecem entre as funções de marketing, logística e produção no ambiente de uma empresa, e dessas mesmas interações entre as empresas formalmente distintas no âmbito do canal de fluxo de produtos.

\section{Quadro Teórico Sobre Inovação}

\begin{tabular}{|c|c|c|}
\hline Conceito de Inovação & $\begin{array}{l}\text { A exploração com sucesso } \\
\text { de novas ideias. }\end{array}$ & $\begin{array}{l}\text { Betz, F. (1997). Managing } \\
\text { Technological Innovation: } \\
\text { competitive advantage from change. } \\
\text { New York: A. Wiley -Interscince } \\
\text { Publication. }\end{array}$ \\
\hline Inovação de Processos & $\begin{array}{l}\text { Consiste em modificações } \\
\text { nos atributos do produto, } \\
\text { com mudança na forma } \\
\text { como ele é percebido } \\
\text { pelos consumidores, }\end{array}$ & $\begin{array}{l}\text { Pennings, (1998). Innovations JM } \\
\text { como precursores de desempenho } \\
\text { organizacional Em: Galliers, R.; } \\
\text { BAETS WRJ (Org.). tecnologia da } \\
\text { informação e transformação } \\
\text { organizacional: inovação } \\
\text { para a organização do século 21.New } \\
\text { York: Wiley. }\end{array}$ \\
\hline Inovação de Produtos & $\begin{array}{l}\text { Afirma que São mudanças } \\
\text { no processo de geração de } \\
\text { produto ou serviço com } \\
\text { impacto no produto final }\end{array}$ & $\begin{array}{l}\text { Rothwell, R. (1994). Para o processo } \\
\text { de inovação de quinta geração. } \\
\text { Ciência Policy Research, v. 1, n. 1, p. } \\
\text { 7-31. }\end{array}$ \\
\hline
\end{tabular}

Fonte: Elaborado pelos autores, 2018. 


\section{Inovação}

A inovação, de acordo com (Betz 1997) tem um conceito bastante amplo e variado o qual depende, principalmente, de sua aplicação. Genericamente, conceitua-se como sendo a exploração com sucesso de novas ideias.

Trata-se de um processo que busca transformar novas ideias em oportunidades e colocálas em prática, bem como o ato ou efeito de tornar algo novo (Tidd; Bessant; Pavitt, 2005, p. $13)$.

Segundo (Damanpour 1991), existem várias possibilidades e formas de inovar, dentre as quais aquelas que se referem a inovações de produtos ou de processos são reconhecidas como inovações tecnológicas, de e para novos mercados, novos modelos de negócio, novos processos e métodos de gestão organizacional. Ou até mesmo novas fontes de suprimentos. Também para (Drucker 1974), a inovação passou a ser reconhecida como fator indispensável para a competitividade e passou a ser incluída na agenda estratégica de muitas empresas.

Apresentando sua colaboração sobre o assunto e concordando com os autores anteriormente mencionados (Braglia, Carmignani, Zammori 2006), afirmam que a inovação representa um importante recurso para a sobrevivência das organizações e apresenta-se como elemento chave para o sucesso de uma empresa em um mercado competitivo.

Dentro desta perspectiva é possível afirmar que as empresas de sucesso são aquelas que de forma consistente criam novos conhecimentos, tecnologias e produtos, construindo assim um negócio de inovação contínua (Tidd; Bessant; Pavit, 2008, p. 16).

Em se tratando da inovação em processo, de acordo com (Cattani 2006), deve apresentar mudanças significativas na geração dos produtos ou serviços. A inovação em marketing se refere a introdução de novos métodos de promoção do produto, seu design que não altere as suas características em termos de funcionamento, embalagem e precificação, ou seja, na inovação organizacional.

A inovação é fundamentalmente um produto do conhecimento e da criatividade, exigindo planos estratégicos flexíveis, controles financeiros não restritivos e sistemas de reconhecimento das inovações implementadas (Grant, 1988, p.14).

Cabe aos gerentes, conforme (Prahalad \& Hamel 1990, p. 18), criar um clima propício à inovação, desenvolvendo habilidades capazes de gerar competências que permitam à 
organização utilizar seu potencial inovador para o aproveitamento das melhores oportunidades do mercado construindo oportunidades para uma futura geração.

As inovações incrementais são geradas através do aprendizado interno e da capacitação acumulada, ao passo que inovações radicais podem trazer modificações substanciais para a sociedade (Paroline; Visconti, 2003, p. 19).

\section{Tipos de Inovação}

Existem diferentes formas de inovação que podem ser classificadas de várias maneiras. Aqui estão destacadas duas destas visões segundo (Jovane, Westkamper, Williams, 2008):

a) Inovação de produto:

De acordo com (Pennings 1998), consiste em modificações nos atributos do produto, com mudança na forma como ele é percebido pelos consumidores.

b) Inovação de processo:

Segundo (Rothwell 1994), afirma que mudanças no processo de geração de produto ou serviço não gera necessariamente impacto no produto final, mas produz benefícios no processo de produção, geralmente com aumentos de produtividade e redução de custos.

\section{Indústria Calçadista}

Em decorrência da alteração do contexto, tanto global quanto local, a indústria calçadista no Brasil vem passando, nos últimos anos, por um processo de reestruturação.

Sem apresentar um diferencial competitivo, a indústria foi pressionada entre produtos de qualidade similar advindos dos países da Ásia, mais econômicos, assim como pelos produtos italianos de preço mais elevado, porém com melhor imagem no mercado internacional (Braglia, Carmignani, Zammori, 2006, p. 17). 
A interação da indústria calçadista com seus fornecedores, depende do tipo de calçado produzido, com classificação, em geral, em quatro grupos principais de acordo com o material de confecção do padrão de matéria prima utilizada: injetados, sintéticos, couro e têxtil. Os calçados injetados, notadamente os de Policloreto de polivinila - PVC são produzidos em fábricas que necessitam de pouca mão de obra.

\section{Metodologia}

Em alinhamento aos objetivos, o presente trabalho apresenta um estudo de caso único, abordagem qualitativa e coleta de evidências por meio de aplicação de entrevistas realizadas em 16 de outubro de 2017, com gestores de indústrias do ramo de calçados que compõem a cadeia de suprimentos na região do Cariri cearense (Salomon, 1999).

A adoção da técnica de pesquisa qualitativa (Scaletzky, 2008) se justifica na medida em que a mesma permite conhecer melhor a natureza do problema pesquisado, considerando-se que as principais características do método são a imersão do pesquisador no contexto e a perspectiva interpretativa do seu encaminhamento (Kaplan; Duchon, 1988).

Em relação aos objetivos, conforme Yin (2005), o trabalho realizado pode ser considerado como pesquisa aplicada, exploratória, pois objetiva oferecer maior conhecimento do problema, para torná-lo mais explícito, através de levantamento bibliográfico apresentando conhecimentos sobre o assunto pesquisado.

\section{Entrevistas com Gestores de Empresas do Ramo de Calçados sobre Inovação de Produtos e de Processos}

A entrevista foi dirigida aos gestores de três empresas da Indústria de calçados, as quais compõem uma cadeia de suprimentos, sendo que as respostas, por terem apresentado muitas semelhanças, foram condensadas em uma resposta única para cada pergunta, que seguem apresentadas em blocos. 


\section{BLOCO I - Conhecimentos sobre o Conceito de Inovação}

Questão 1 - Com base nas mais diversificadas afirmações que se encontram no mercado, quais são os tipos de inovação de seu conhecimento (Jovane, Westkamper, Williams, 2008)?

Respostas: Inovação vai além do que é vista como uma tecnologia sofisticada. Inovar é considerar diferentes estratégias que podem acontecer em diferentes áreas da organização, tal como, produtos e serviços, canais de distribuição e processos de produção.

Questão 2 - Em seus ambientes de negócios - Fabricação e comercialização de produtos - o que está sendo feito para identificar oportunidades de inovação, (Freeman; Soete, 1997)?

Respostas: Indo às fontes, ou seja, indagando no mercado o que o cliente espera adquirir ou conquistar em termos de novidades para se tornar diferente. Como afirmou Drucker (1974), o cliente raramente compra o que a empresa acha que deve ser vendido. Procura-se um desafio ou um problema importante que não esteja sendo adequadamente resolvido através das soluções atuais apresentadas. Daí procura-se implementar mudanças para que o novo produto apareça para o cliente.

Questão 3 - De acordo com Pennings (1998), quando afirma que as novas ideias e os novos processos levam às organizações ao processo de inovação, como você faz para encontrar uma boa ideia para implementar inovações?

Resposta: Lembre-se da frase de Pablo Picasso: "Bons artistas copiam, grandes artistas roubam". Procura-se tomar emprestado ideias de outras indústrias e até mesmo de outros setores.

\section{BLOCO II - Inovação nos Produtos}

Questão 4 - Para uma boa apresentação de produtos na oportunidade de se prospectar um cliente é necessário que se tenha um bom esboço do produto através de um potfólio, gerando 
assim um melhor desempenho em sua produção e venda. O que significa para você um desempenho "suficientemente bom" (Betz 1997)?

Resposta: Significa um desempenho acima do mínimo esperado, para resolver de forma adequada um problema ou para atender adequadamente à necessidade de um cliente, sacrificando o desempenho ao longo das dimensões tradicionais, que com isso pode abrir novos caminhos para inovar, podendo adotar práticas de "trade-off" ou de escolhas conflitivas, buscando soluções de compromissos, oferecendo novas formas que introduzam novas formas de benefícios relacionados a simplicidade e acessibilidade.

Questão 5 - O gestor tem necessidade de auto avaliação constante no mercado, para que se veja como estar perante a clientela e sua concorrência (Benchmarking). Como você avalia se sua ideia é adequada para o que se propõe a fazer (Paroline; Visconti, 2003)?

Resposta: Procura-se validar as ideias com padrões que reflitam as reais necessidades dos clientes, lembrando-se da frase de Scott Cook (Criador de um famoso software para contabilidade financeira): "para cada falha que nós tivemos também tínhamos planilhas de cálculos que pareciam incríveis". Sendo assim, implemento várias experiências de alto retorno sobre os investimentos, para tentar resolver situações que são consideradas críticas.

Questão 6 - Toda empresa ao iniciar suas atividades deve estabelecer um horizonte temporal para atingir seus limites de satisfação. Quanto tempo levou para seu negócio ganhasse escala, em termos de inovação (Grant, 2003)?

Resposta: Quase sempre mais do que as projeções iniciais, considerando que você deve ser paciente para a necessidade de crescimento e impaciente para a necessidade de obter lucros, observando a "nova norma" de mudar constantemente que exige domínio de "transformação constante" e contínua.

Questão 7 - É importante que a empresa reconheça seu grau de dificuldade para que possa superá-la. Qual a dificuldade maior encontrada para a inovação (Kotler; Keller, 2006)?

Resposta: A grande maioria das organizações foram projetadas e estruturadas para execução, não para inovação. Então o empresário (gestor) deve se preparar para punir qualquer 
coisa que apresente sinais de fracasso e reconhecer que o fracasso é muitas vezes uma parte crítica do processo de inovação.

Questão 8 - Para que se possa atingir um satisfatório grau de inovação e necessário que se utilize de estratégias eficientes nos processos. Quais são os elementos que compõem as estratégias de inovação em suas empresas. (Braglia, Carmignani, Zammori, 2006)?

Resposta: Definição clara das metas, um portfólio de projetos ou iniciativas de inovação, mecanismos para alocar recursos que gerem condições para executar bem o portfólio e a definição clara das áreas de foco para a inovação.

Questão 9 - O nível de direção da empresa representa o planejamento e a tomada de decisões no negócio. Qual o principal papel desempenhado pelos altos executivos das empresas em relação ao processo de inovação (Kotler; Keller, 2006)?

Resposta: A organização no dia-a-dia e muita habilidade para conduzir o processo na prática.

Questão 10 - Muitos são os negócios que se acomodam e acham que já atingiram sua capacidade, sendo que podem estar com capacidade ociosa e demanda reprimida. De acordo com Segundo Rothwell (1994), você considera que já conseguiu alcançar a capacidade de inovar em "larga escala"?

Resposta: Algumas vezes até já tive essa sensação, porém quando faço uma avaliação mais criteriosa observo que ainda estou dependendo de mecanismos que me protejam contra as armadilhas do modelo tradicional de negócio.

Questão 11 - O processo de inovação é considerado contínuo e ininterrupto. Em relação ao número de novos processos produtivos introduzidos nos últimos anos em sua empresa, o que você tem a informar (Rothwell (1994)?

Resposta: Procuro sempre inovar os nossos processos, investindo em máquinas, equipamentos e mão de obra constantemente atualizadas, para que possa alcançar o máximo de satisfação de nossos consumidores, sem perder de vista o controle de custos e utilizando o que é possível de mais moderno dentro das tecnologias de produção.

\section{Id on Line Rev. Mult. Psic. V.12, N. 42, p. 1143-1160, 2018 - ISSN 1981-1179} Edição eletrônica em http://idonline.emnuvens.com.br/id 
Questão 12 - Para encerrar, que mensagem você deixaria sobre esse assunto tão importante na indústria de calçados (Kotler; Keller, 2006)?

Resposta: Quanto ao processo: Adotar um modelo de gestão bem definido e direcionado a adequação dos processos, de acordo com o que há de mais moderno no mercado.

Quanto ao Produto: $\mathrm{O}$ alinhamento constante com os anseios do mercado consumidor é o mais importante para que o produto seja um meio de satisfação das necessidades de quem o utiliza, em qualquer das suas dimensões (evitar o desconforto, prazer, senso de realização pessoal, status, reconhecimento e destaque).

\section{Análise dos Resultados e Considerações Finais}

A análise qualitativa é apresentada nesta subseção sobre o nível que cada uma das questões obteve. Em seguida, as observações gerais para cada um dos itens.

As entrevistas permitiram coletar dados específicos, além de apresentar uma visão ampla e detalhada do nível de maturidade da gestão da inovação das empresas.

Abaixo se apresentam as interpretações das afirmações dos gestores referentes a cada um dos questionamentos levantados.

\section{Questão 1}

As empresas não possuem um setor de PDI (Pesquisa, desenvolvimento e inovação), porém visualizam a necessidade de implantar, pois enxergam como um passo no sentido de desenvolver melhor os tipos de inovação, os quais afirmam conhecer.

\section{Questão 2}

Várias inovações já foram realizadas e já tiveram vários projetos de inovação. Atualmente há um processo definido para inovação no produto, bem como para melhorar seus processos. Os gestores afirmam que as oportunidades são avaliadas periodicamente.

Justificando a resposta, afirmam que atualmente as empresas conseguem atingir o nível bom de inovação em se tratando de uma abordagem focada no produto e no processo. Entretanto, uma abordagem da organização como um todo, pode não refletir o mesmo estágio. 


\section{Questão 3}

Buscando sempre ideias novas reconhecem que há uma metodologia para os processos, e também, processos já automatizados. Já no que se refere a produtos, cada alteração no produto para garantir a integridade dos cálculos dos custos apresentam uma série de fragilidade.

\section{Questão 4}

Os entrevistados explicam que no momento o foco é está voltado para a inovação nos produtos e nos processos, e ainda não na organização como todo, mas que estão investindo na ideia e, receberão em breve consultorias, para saberem como podem inovar, não só em produtos e processos, mas de modo geral.

\section{Questão 5}

Há um repositório centralizado para as ideias e estas, são registradas por um responsável. As ideias são classificadas e o acesso é compartilhado por colaboradores. Justificando a resposta, o banco de ideias é sim, avaliado pelos gestores.

\section{Questão 6}

Afirmaram os gestores que o processo de busca pela inovação é constante e a implementação vem acontecendo em todo o tempo de existência das empresas. Porém ainda estão em um período de maturação e em termos de crescimento em escala ainda não são considerados tão firmes nesse propósito.

\section{Questão 7}

Na situação atual a empresa é incapaz de medir os custos e o retorno sobre os investimentos gerado pelas práticas de inovação. Justificando a resposta, os resultados das inovações são avaliados de forma subjetiva. Em algumas situações é possível identificar a redução de custos. É mais fácil perceber o que ela deixou de pagar do que aquilo que recebeu com a inovação. 


\section{Questão 8}

As empresas conseguem identificar as técnicas. Existem padrões definidos e as informações são compartilhadas. Há uma metodologia para o processo, e também, processos já automatizados. As alterações que são feitas no produto são oriundas de sugestões de inovação. Justificando, existem então, metodologias para apoio ao processo e a automação é identificada em algumas partes, porém, ainda não em todas as etapas.

\section{Questão 9}

Existem algumas pessoas diferenciadas dentro da organização, as quais as empresas conseguem identificar com visão de futuro e destina cargo para essas pessoas, através de nomeações para os cargos de executivos. No cenário atual, o analista de negócios é considerado um visionário. Ele recebe incentivo para assistir a palestras e para buscar fontes de informações e inovações externas.

\section{Questão 10}

Inovação em larga escala é um patamar desejado, porém em alguns momentos eles até já imaginam ter atingido, só que ao mesmo tempo se dão conta de que ainda estão presos a algumas práticas tradicionais que os impedem de chegar a tal resultado.

\section{Questão 11}

Quanto aos processos produtivos com moldes inovados os mesmos afirmam que tem introduzido constantemente, mas reconhecem que ainda há muito o que fazer para atingir o objetivo traçado para as indústrias de uma forma geral.

\section{Questão 12}

Os gestores e executivos das empresas pesquisadas têm um pouco de conhecimento do que está no Manual de OSLO que afirma o seguinte: para haver inovação de produto é necessária a introdução de melhoria significativa nas características do produto (bem ou serviço), por exemplo, com melhora em especificações técnicas, componentes e materiais.

Esse tipo de inovação altera as características funcionais do produto. Já para a inovação de processo pressupõe um método de produção ou distribuição novo ou significativamente 
melhorado, por exemplo, como a introdução de novos equipamentos de automação em uma linha de produção ou novos métodos de distribuição. Esse tipo de inovação altera as características de produção/distribuição, mas não altera necessariamente características funcionais do produto.

\section{Conclusões}

A principal conclusão do estudo é que as práticas de inovação adotadas pelas empresas na busca pela inovação dos produtos e processos não são suficientes para torná-las mais competitivas, deixando uma lacuna entre as práticas ideais segundo a literatura e as utilizadas por seus gestores.

Conclui-se também que de forma geral os gestores entrevistados confundem os conceitos de inovação e aplicam de forma equivocada tornando-os menos competitivos do que se formassem cluster, conforme orienta (Bengtsson e Kock 2000).

Aponta-se como a principal razão para esta ausência é que algumas práticas adotadas são somente com o objetivo de redução de custos e maior volume de venda.

A ausência de um requisito estratégico para empresas cujos produtos competem pela diferenciação, está comprometendo o resultado alcançado na região do Cariri Cearense, o que confirma a hipótese levantada para investigação.

Portanto, as empresas devem rever seu posicionamento no mercado, procurando formar clusters e utilizar-se do conceito de coopetition.

A inovação sistematicamente planejada deve ter início através da análise das oportunidades, porém não é convenivente se negar que tais fontes apresentarão importância distinta em diferentes períodos. É iperioso no entanto não ignorar que os possíveis inovadores necesswitam dar toda atenção que o mercado necessita, lembrando que a inovação surtir eficácia deve ser implementada de forma simples e centralizada, especifica, e que apresenta um nível de exigência elevado com relação ao conhecimento e persistência.

Assim, podemos afirmar que a inovação para fazer a comunicação de produtos e serviços é um imperativo para sobrevivêcia em um mercado muito competitivo. Torna-se 
também fundamental levar-se em conta que, uma das grandes fontes de inovação é hoje o marketing.

Gerir a inovação implica lidar com um interessante paradoxo. Por um lado, requer uma boa dose de conhecimento técnico. Mas, por outro lado, quanto mais se sabe, mais possibilidades há de se deixar se envolver por esse know-how acumulado.

Para as futuras pesquisas, sugerimos outros estudos de caso aprofundados sobre o assunto, procurando construir um banco de dados para conclusões robustas, inclusive enveredando para uma pesquisa quantitativa.

Sugerimos também uma pesquisa nas indústrias sobre o tema em estudo, incluindo um maior número de empresas, cujos dados serão analisados por meio de técnicas de estatística multivariada.

\section{Referências}

Abdulmalek, F, Rajgopal, J: (2007).Analisando os benefícios de fabricação magra e mapeamento de fluxo de valor por meio de simulação: Um estudo de caso setor processo. Jornal Internacional de Produção Economia 107, 223-236.

Achanga, P., Shahab, E., Roy, R., Nelder, G: (2006). Fatores críticos de sucesso para implementação lean nas PME. Journal of Manufacturing Tecnology Gestão 17 (4), 460-471

Baskerville, R. L. Wood-Harper, A. T. A critical perspective on action research as a method for information systems research. Journal of Information Technology, v. 11, n. 3, p. 235-246, set. 1996.

Betz, F. (1997). Managing Technological Innovation: competitive advantage from change. New York: A. Wiley -Interscince Publication.

Boer, Cr, Dulio, S: (2007). Personalização de massa e calçado: Mito, salvação ou Realidade. Springer, Heidelberg.

Braglia, M., Carmignani, G., Zammori, F: (2006). A nova abordagem de mapeamento de fluxo de valor de sistemas de produção complexas. International Journal of Production Research 44 (18-19), 3929-3952. 
Carpanzano, E., Cataldo, E: (2003). Um design modular e Verificação Baseada em Simulação do Código Logic Control para uma sapata de Agile Manufacturing System: Proc. Industrial Conference Simulação, ISC 2003, a EUROSIS, Valência, Espanha, 09-11 junho (2003).

Campos, E. M. (2016). Os Dois Lados da Moeda: A sobrevivência e mortalidade dos Negócios. EDITORA IFPB. João Pessoa.

Cattani, K.; Gilland, W.; Heese, H. S. (2006). Pricing strategies for a manufacturer adding a direct channel that competes with the traditional channel. Production and Operations Management, v. 15, n. 1, p. 40-56.

Cooper, R. G.(2001) Winning at new products. Accelerating the process from idea to launch.3th ed. Addison : Wesley Publishing Company.

Damanpour, F. (1991). Inovação organizacional: uma meta-análise dos efeitos dos determinantes e moderadores. Academy of Management Journal. v. 34, n. 3, p. 555-590.

Drucker, P. F. (1974). O Gerente Eficaz. Editora Zahar.

Fajnzylber, F. (1998). Competitividad Internacional: evolucion e lecciones. Revista de la Cepal..

Freeman, C; Soete, L. (1997). Economics of Industrial Innovation. ED. Routledge, $3^{\text {a }}$ edição. Galbraith , J. R.; Lawler, (1995). E. Organizando para competir no futuro -Estratégias para gerenciar o futuro das organizações. São Paulo : Makron Books.

Grant, R. M. (1988) Contemporary strategy analysis. 3th ed. Malden : Blackwell.

Gunasekaran, R: (1999). Agile Manufacturing: uma estrutura para pesquisa e desenvolvimento. Jornal Internacional de Produção Economics 62, 87-105.

Jones, Dt, Hines, P., Rich, N: (1997). "logística lean”. Jornal Internacional de Distribuição Física e Gestão Logística 27 (3/4), 153-173.

Jovane, F., Westkämper, E., Williams, D: (2008). O Manufuture Estrada: para Competitivo e Sustentável de alta Adicionando-Value Manufacturing. Springer, Heidelberg.

Kaplan, B.; Duchon. D. (1998). Combining quantitative and qualitative methods in informations systems research: a case study. Mis Quarterly. 
Klaesener, D; Appio, J. Importância da atividade importadora. Disponível: < http://revistas.utfpr.edu.br/pb/index.php/SysScy/article/view/266>. Acesso em: 15.08.2017 Kotler, P.; Keller. K.L.(2006). Administração de marketing .12a edição. São Paulo: Pearson Prentice Hall.

Paroline, C.; Visconti, F. Footwear manufacturing districts: the effects of internazionalisation, delocalization and new technologies. Paper apresentado na onferência Clusters, Industrial Districts and Firms: the Challenge of Globalization, Università degli Studi di Modena e Reggio Emilia, set. 2003. Disponível em: . Acesso m: 21.08.2017.

Pennings, (1998). Innovations JM como precursores de desempenho organizacional. Em: Galliers, R.; BAETS, WRJ (Org.). tecnologia da informação e transformação organizacional: inovação para a organização do século 21. New York: Wiley.

Prahalad, C. K.; Ramaswamy, V. (2004). O futuro da competição: como desenvolver diferenciais inovadores em parceria com os clientes. Rio de Janeiro: Elsevier.

Rogers, E. M. (2003). Diffusion of Innovation $5^{\text {a }}$ edição. Nova Iorque: Free Press.

Rother, M., Shook, J: (2003). Aprendendo a ver: Value Stream Mapping para agregar valor e eliminar desperdícios. O Instituto Lean Enterprise.

Rothwell, R. (1994). Para o processo de inovação de quinta geração. Ciência Policy Research, v. 1, n. 1, p. 7-31.

Salomon, D. V. (1999(. Como fazer monografia 9. ed. São Paulo: Martins Fontes.

Scaletzky, C. (2008). Pesquisa aplicada/pesquisa acadêmica: O caso Sander. In: Congresso Brasileiro de Pesquisa e Desenvolvimento em Design. São Paulo: Centro Universitário Senac.

Schmitz, E. (2000). Fundamentos da Didática. $7^{\text {a }}$ Ed. São Leopoldo, RS: Editora Unisinos.

Schumpeter, J. (1942). Capitalismo, socialismo e democracia. Rio de Janeiro: Fundo de Cultura, $1961.1^{\text {a }}$ edição.

Schumpeter, J, (1984). "Towards a strategic theory of the firm", in Lamb, R.B. (org.), Competitive Strategic Management, Englewood Cliffs, NJ: Prentice Hall.

Setht, D., Gupta, V: (2005). Aplicação de mapeamento de fluxo de valor de operações magras e redução do tempo de ciclo: um estudo de caso Indiana. Prodution Planeamento e Controlo 16 (I), 44-59. 
Sharifi, H., Colquhoun, G., Barclay, I., Dann, Z: (2001). Agile fabricação: a gestão e enquadramento operacional. In: Anais do Institution of Mechanical Engineers, vol. 215-B, pp. 857-869.

SEBRAE - Serviço Brasileiro de Apoio às Micro e Pequenas Empresas, (2016). Comece Certo a Indústria de Calçados. 2016.

SINDINDÚSTRIA - Sindicato das Indústrias de Calçados e Vestuários de Juazeiro do Norte Ceará. Informativo Bimestral - 02/2017.

Tidd, J.; Bessant, J.; Pavitt, K. (2008). Gestão da Inovação. $3^{a}$ edição. São Paulo: Bookman.

Tidd, J .; Bessant, J .; Pavitt, K. (2005). Gestão da inovação: integração tecnológica, de mercado e mudança organizacional. New York: John Wiley \& Sons.

Westkamper, E. (2007). Desenvolvimento Estratégico de fábricas sob a influência de tecnologias emergentes. Anais da CIRP 56-1, 419-4225.

Yin, R. K. (2005). Estudo de caso: planejamento e métodos. $3^{\circ}$ edição. Porto Alegre: Bookman 2005.

\section{Como citar este artigo (Formato ABNT):}

BELÉM, José de Figueiredo; LIMA, José Eduardo de Carvalho; SOUSA, Josiano Cesar de; ALVES, Murilo Barros. A Gestão da Cadeia de Suprimentos e o Nível de Inovação: Estudo de Caso nas Indústrias de Calçados na Cidade de Juazeiro do Norte - Ceará . Id on Line Rev.Mult. Psic., 2018, vol.12, n.42, p. 1143-1160. ISSN: 1981-1179.

Recebido: 28/06/2018;

Aceito: $30 / 10 / 2018$ 